\title{
Amino acid enrichment and compositional changes among mammalian milk proteins and the resulting nutritional consequences
}

\author{
Nora Khaldi, ${ }^{* 1}$ Thérèse A. Holton, ${ }^{\star}{ }^{1}$ and Denis C. Shields ${ }^{\star 2}$ \\ *UCD Conway Institute of Biomolecular and Biomedical Research, School of Medicine and Medical Sciences, \\ UCD Complex and Adaptive Systems Laboratory, and \\ †Food for Health Ireland, University College Dublin, Dublin 4, Ireland
}

\section{ABSTRACT}

Milk is a hallmark of mammalian evolution: a unique food that has evolved with mammals. Despite the importance of this food, it is not known if variation in AA composition between different species is important to milk proteins or how it might affect the nutritional value of milk. As milk is the only food source for newborn mammals, it has long been speculated that milk proteins should be enriched in essential AA. However, no systematic analysis supports this assumption. Although many factors influence the overall nutritional value of milk, including total protein concentration, we focused here on the AA composition of milk proteins and investigated the possibility that selection drives compositional changes. We identified 9 major milk proteins present in 13 mammalian species and compared them with a large group of nonmilk proteins. Our results indicate heterogeneity in the AA composition of milk proteins, showing significant enrichment and depletion of certain AA in milk-specific proteins. Although high levels of particular AA appear to be consistently maintained, orthologous milk proteins display significant differences in AA composition across species, most notably among the caseins. Interspecies variation of milk composition is thought to be indicative of nutritional optimization to the requirements of the species. In accordance with this, our observations indicate that milk proteins may have adapted to the species-specific nutritional needs of the neonate.

Key words: amino acid composition, amino acid enrichment, milk protein evolution, milk nutrition

\section{INTRODUCTION}

Milk, secreted by the mammary glands of females, is a hallmark of mammalian evolution. Known benefits of milk include its rich macro- and micronutrient content

Received July 30, 2013.

Accepted November 30, 2013.

${ }^{1}$ These authors contributed equally to the study.

${ }^{2}$ Corresponding author: denis.shields@ucd.ie and various health-promoting roles. Milk is primarily composed of 3 parts: the protein-rich whey, the casein micelles that transport calcium and give milk its white color, and the milk fat globule (Jensen, 1995). Milk secretion has been conserved for approximately 218 million years (Meredith et al., 2011), from platypus to modern human, as a means of nutrition for the newborn.

Five small milk proteins are strikingly "milk specific": the whey protein $\alpha$-LA and the 4 caseins $\left(\alpha_{\mathrm{S} 1}-\mathrm{CN}\right.$, $\alpha_{S_{2}}-\mathrm{CN}, \beta-\mathrm{CN}$, and $\left.\kappa-\mathrm{CN}\right)$, which are synthesized in the mammary gland during pregnancy or at the onset of lactation (Burditt et al., 1981). These proteins are recognized as being the most abundant proteins in the milk of many species, including human and bovine milks (Lemay et al., 2009). Milk also contains many other proteins at lower frequency, such as lactoferrin, which is associated with whey, and mucin, lactadherin, butyrophilin, and xanthine dehydrogenase, all of which are associated with the milk fat globule (MFG). These 5 larger milk proteins are not specific to milk, belonging to families of proteins that can also be found outside the mammary gland and whose synthesis is not dependent on pregnancy and lactation (Zotter et al., 1988; Parry et al., 1992; Valenti and Antonini, 2005; Véron et al., 2005). Nine of these relatively abundant milk proteins are present in at least 9 of 13 representative species across Mammalia for which genome sequences are available (i.e., human, chimp, monkey, mouse, rat, guinea pig, rabbit, cat, dog, horse, cow, opossum, and platypus; see Table 1).

Mammals differ greatly in the nutritional contents of their milks. For example, the composition and, in particular, protein concentration of milk varies greatly among species. Of those species extensively studied to date, an interspecies difference in protein concentration ranges from approximately 1 to $20 \%$, and is believed to reflect species-specific optimization to the nutritional requirements of the neonate (Jenness, 1974b; Hambræus and Lönnerdal, 2003; Martin et al., 2013). Further important variation is seen with respect to whey protein-to-casein ratios in milks. In humans, the 
typical whey-to-casein ratio during lactation is approximately 60:40, but can fluctuate from 80:20 to 50:50 during early and late stages of lactation, respectively (Lönnerdal, 2003). However, in the majority of mammalian milks, the converse of this is observed, with the total proportion of casein summing to more than that of whey (Jenness, 1974a, 1986).

Within the caseins alone, which are important transporters of calcium phosphate, significant disparity can be observed among species. For instance, human milk contains only 3 caseins, with bovine and murine milks containing 4 and 5, respectively (Bevilacqua et al., 2006). Moreover, the individual proportion of each casein protein contrasts considerably in mammals. In human milk, $\beta-\mathrm{CN}$ is the principal component of the casein micelle, whereas in rabbits, $\alpha_{\mathrm{S1}^{-}} \mathrm{CN}$ dominates, with equivalent ratios of both caseins found in murine, porcine, and bovine milks (Martin et al., 2003). It is thus clear that the species-specific adaptation of milk to the nutritional demands of their young is a multifactorial process.

The role of AA composition of milk proteins in conferring optimized nourishment across mammals is less established. It is commonly believed that proteins from the mother's milk meet the AA needs of the newborn, especially the requirement of EAA not naturally produced by mammals (e.g., for human: His, Ile, Leu, Lys, Met, Phe, Thr, Trp, and Val; and in the case of premature infants, Cys and Tyr; Reeds, 2000; Fürst and Stehle, 2004). This assumption is supported by the fact that milk constitutes the only food source for mammalian neonates and thus should provide the essentials for growth and development beyond those stores established by the offspring in utero.

Food composition and nutrition tables established by the International Network of Food Data Systems (INFOODS), which is part of the Food and Agriculture Organization (FAO) of the United Nations (http:// www.fao.org), show that milk-specific proteins, especially whey proteins, are rich in infant EAA compared with other food sources. For example, the whey protein $\alpha-\mathrm{LA}$ is shown to be rich in Thr, a precursor of serotonin, which is used as a supplement to relieve sleep disorders (Fernstrom, 1983; Schaechter and Wurtman, 1990). Further to this, whey proteins have higher frequencies of branched-chain AA (Val, Ile, and Leu) than many other food products (Davis et al., 1994; Hui et al., 2007). As a result, whey is used as a supplement for active individuals such as athletes (for a review, see Ha and Zemel, 2003). Surprisingly, to date it has not been shown if certain AA are enriched in milk proteins compared with the rest of the proteome.

Because mammalian species differ in many morphological, physiological, and behavioral traits, we set

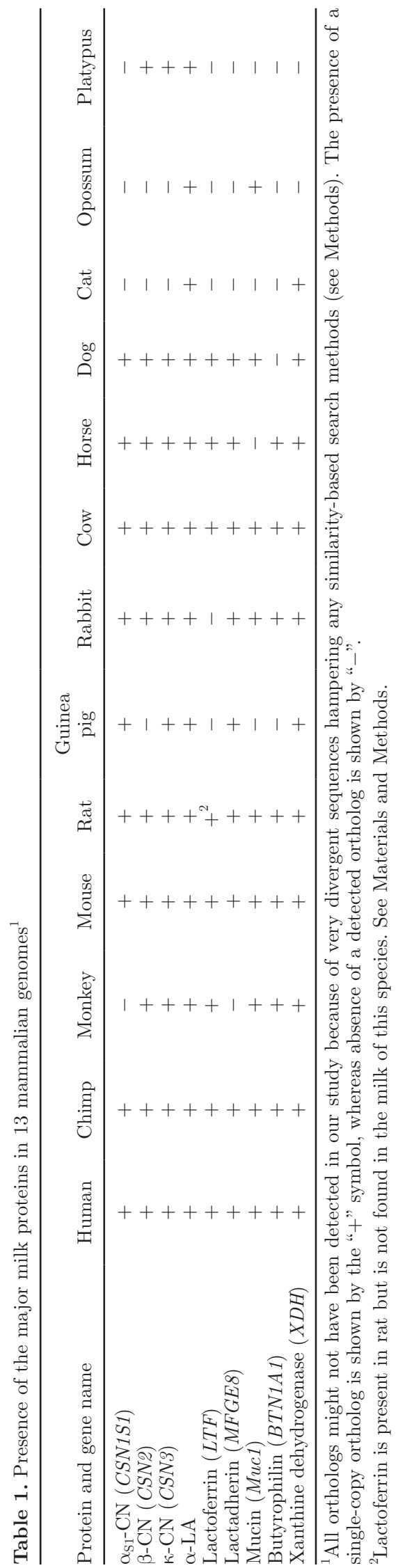

Journal of Dairy Science Vol. 97 No. 3, 2014 
out to determine if evidence also exists for important changes in AA composition. It has been already shown that milk proteins exhibit significant levels of change in their charge and isoelectric point, and that this is most likely due to functional divergence (Khaldi and Shields, 2011). Some species might require higher quantities of certain types of AA because of a species-specific trait that translates into the need for a certain AA (Nelson and Evans, 1958; Snyderman et al., 1961).

Here, we compare milk proteins and nonmilk proteins and address 2 questions: (1) is the AA composition of milk proteins conserved between different mammalian species? (2) Are milk proteins enriched in certain AA and which ones in particular? We also specifically compare human to bovine milk proteins in terms of their AA compositions.

\section{MATERIALS AND METHODS}

The human, chimp, monkey, mouse, rat, guinea pig, rabbit, cat, dog, horse, cow, opossum, and platypus protein sequences were downloaded from various sequence databases (see Supplemental Table S2 for a detailed list of sources; http://dx.doi.org/10.3168/jds.2013-7317). The major milk proteins (conforming to the nomenclature outlined in Farrell et al., 2004) were selected on the basis of their presence in at least 9 mammalian species out of the 13 (see Table 1). In addition, the 9 species needed to include human, chimp, cow, and mouse. This condition was imposed to simplify our analysis of selective constraints in milk composition (see below). The identified proteins also represent the 3 parts of milk: whey, casein, and MFG. We used the 9 major milk proteins defined in human and cow to detect their orthologs in the 13 other genomes, by reciprocal BLAST hits (Altschul et al., 1997). We considered as orthologs all proteins that satisfied 78 reciprocal hits (if the protein belongs to 13 species). For some milk proteins, we could not detect an ortholog in all 13 mammals because of very divergent sequences, which hampered any similarity-based search methods.

\section{Orthologs and Signal Peptides}

To find orthologs, we identified 13-way mutual best BLASTP (Altschul et al., 1997) hits among human, chimp, monkey, mouse, rat, guinea pig, rabbit, rat, dog, horse, cow, opossum, and platypus. This method resulted in 1,412 sets of putative orthologs among the 13 species. Each 13-set of proteins was aligned using ClustalW (Thompson et al., 1994). Because milk proteins lose their signal peptide before entering the milk, we recursively implemented the SignalP software (Petersen et al., 2011) on each of the proteins to remove the signal peptide. This was additionally carried out on all nonmilk proteins.

\section{Ancestral Types of Milk Protein}

To examine species-specific compositional differences in the 13 mammals, we reconstructed the ancestral proteins of each of the milk proteins by first aligning the orthologs of each of the 9 milk proteins using ClustalW (Thompson et al., 1994). This was followed by a maximum likelihood reconstruction using codeml from the paml package (Yang, 2007). To avoid biased ancestral sequences, we manually curated the alignments to remove the sections of the alignments that appeared to be a species-specific gain of a large region of protein (an example of this would be $\beta$-CN, which has a long insertion in rodents). Standard ancestral reconstruction is performed in such a way that it incorporates all AA positions, even those that are found in only one species. To avoid this, we manually removed positions that were only found in one species and not in the others from the ancestral sequences.

For $\alpha$-LA, we removed positions 14 and $15(\mathrm{NH})$, which are only found in opossum. For the same reason, we removed position $111(\mathrm{Q})$. We also removed positions 127 to 143 (GAPALVVPALNSETPVP) in the rat because this region is unique to that species. For $\beta-\mathrm{CN}$, we removed positions 46 to 49 (LPTT) and 114 to 117 (PQQN), which are only found in platypus.

\section{AA Conservation}

We determined for each AA the 6 pairwise differences of frequencies between the orthologs in human, chimp, cow, and mouse. The sum of the 6 pairwise differences was considered a measure of the quantity of conservation of one protein between these 4 species for each AA individually. When we considered a group of more than one protein, the measure became the median of all the measures found for each protein individually. We used this approach on the 4 groups in milk ( $\alpha$-LA, caseins, lactoferrin, and nonmilk proteins). We compared these values with those calculated for the nonmilk proteins. We used the Mann-Whitney test on the median of the milk group and the nonmilk group to assess significance. Here, the problem of multiple testing, arising from the use of $20 \mathrm{AA}$, was corrected for using the Bonferroni correction.

\section{AA Composition and Enrichment in Human Milk}

To investigate differences in milk AA composition, we compared the frequencies of each $\mathrm{AA}$ in the milk proteome of human to that of the set of all orthologous 
nonmilk proteins in the human proteome. We used the Mann-Whitney test to assess significance. Again, we corrected for multiple testing using the Bonferroni correction, where $\mathrm{n}=20$.

\section{AA Composition Differences Between Human and Cow Milk}

To investigate differences in milk AA composition between human and cow, we compared the frequencies of each AA in human to that of its ortholog in cow. We used the Mann-Whitney test to assess significance and corrected for multiple testing using the Bonferroni correction, where $\mathrm{n}=20$.

\section{RESULTS AND DISCUSSION}

As milk proteins are found in different concentrations and are associated with different constituent parts of milk (i.e., whey, caseins, or MFG), to conduct a fair analysis, we subdivided the major milk proteins into 4 groups. The first 2 groups, which represent the milkspecific proteins, are (1) the caseins, and (2) the whey protein $\alpha$-LA (with both groups tending to be present in high concentrations in milk; Bordin et al., 2001). The other 2 groups, representing non-milk-specific proteins, are (3) proteins associated with milk fat globules (i.e., butyrophilin, lactadherin, mucin, and xanthine dehydrogenase), and (4) the lactoferrin protein found in whey.

We used the Wilcoxon test to establish whether a significant difference existed between our observations. Here, we were interested not only in the significance of a result, but also in the level of change of an AA, irrespective of significance. Indeed, even though the change may not be significant, it is still a good indicator of which AA are most enriched. Hence, all figures include Bonferroni-corrected $P$-values when they were significant (represented in figures by the symbol + , where $\mathrm{n}=20$ ), as well as the significant values before correction (indicated by ${ }^{*}$ in figures). When we refer to observations that were significant only before Bonferroni correction, it is important to note that we were not accounting for multiple comparisons.

\section{Species-Specific Variation in the AA Composition of Individual Milk Proteins}

To analyze the AA composition of milk proteins and determine possible species- and lineage-specific enrichments, we reconstructed the ancestral sequence of 2 major milk proteins - $\alpha-\mathrm{LA}$ and $\beta-\mathrm{CN}$ - to allow for the prediction of ancestral AA frequencies (Figure 1; Supplemental Table S1; http://dx.doi.org/10.3168/ jds.2013-7317). Essentially, the computer program co- deml estimates the most likely state of the common ancestor of two or more species (at the point where the branches join in the tree) by working backward from the sequences observed in present-day species. Reconstructions were carried out under a maximum likelihood framework based on AA substitution models (Yang et al., 1995) implemented in codeml (Yang, 2007; see Methods).

$\alpha$-Lactalbumin represents one of the most abundant milk proteins, and therefore even individual AA changes in this protein could alter the nutritional qualities of milk. Aside from Cys, which has remained constant through the evolution of $\alpha-\mathrm{LA}$, most likely because of its structural role in disulfide bond formation (Shewale et al., 1984; Permyakov and Berliner, 2000), several AA have experienced substantial enrichment or depletion (Figure 1). Amino acids displaying considerable enrichment at various points in evolution are Thr (in rabbit); Ala, Val, and Pro (in rat); Glu (in the rodent ancestor); and Gly (in dog). Those notably depleted are Ala (in horse); Val and Glu (in primates); Pro (in cat and opossum); Thr and Gly (in guinea pig); and Trp (chimp).

Since the divergence of opossum from the other 11 species, there appears to have been a shift in Asn and His, such that their frequency is greatest in the opossum (Figure 1). Rat $\alpha$-LA is richest in Val $(5.7 \%$, compared with primates, who had a much lower frequency of $1.6 \%$ ). Aside from rat and mouse, in general, we did not observe dramatic changes between closely related species, with divergence in AA composition typically increasing with phylogenetic distance (Figure 1). Thus, although the EAA Ile is found at a higher frequency in primates than in cows (11.5 vs. $6.5 \%)$, the shift in composition appears to have occurred gradually (Figure 1). It is interesting that although none of the EAA were completely missing from this protein, the same was true for the NEAA. This is despite the fact that $\alpha-\mathrm{LA}$ is a short polypeptide ( $\sim 140$ residues), so short that there was insufficient statistical signal to determine if the above changes in frequency were significant. It is unclear whether AA showing compositional changes may affect the functional role of $\alpha$-LA in lactose production because it is hard to measure the effect of such variation on specific functionalities.

$\beta$-Casein also showed several species-specific frequency variations (see Supplemental Table S1; http:// dx.doi.org/10.3168/jds.2013-7317). In fact, this protein displayed a 2-fold change in frequency for 11 AA in 11 species (Supplemental Table S1). Similar to what was seen in $\alpha$-LA, none of these changes were predominantly among the EAA (Figure 1; Supplemental Table S1). $\beta$-Casein has evolved rapidly, potentially giving rise to dramatic changes in AA frequencies. A considerable range of frequency of Trp was observed, extending from 


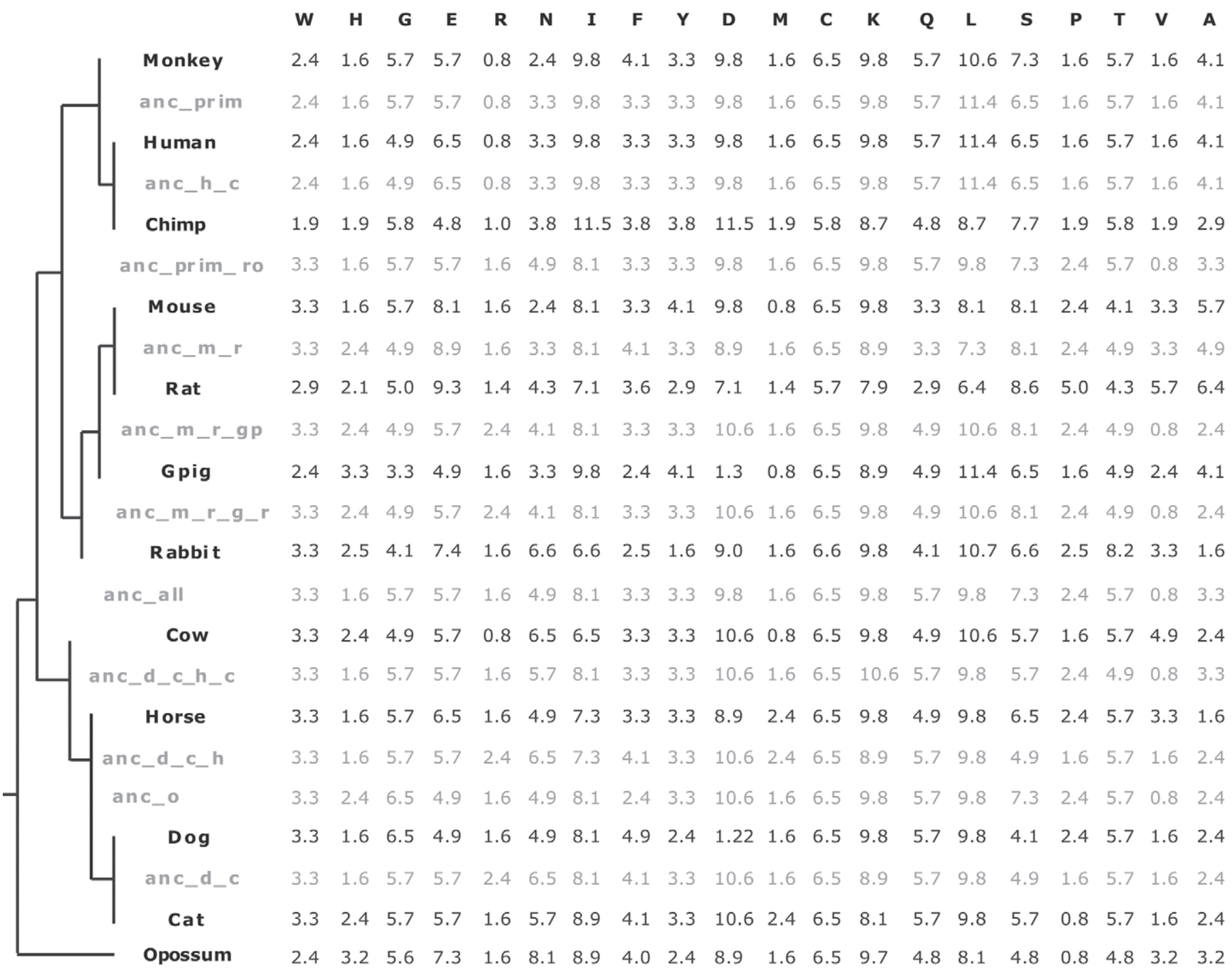

Figure 1. Amino acid frequencies of $\alpha$-LA in 13 mammalian species and their predicted ancestors. The species phylogeny is from Benton and Donoghue (2007). Extant species are shown in black and predicted ancestors are shown in gray.

$0 \%$ in rodents to $5 \%$ in primates (Supplemental Table S1; http://dx.doi.org/10.3168/jds.2013-7317), because of the relatively sudden gain of this AA in the primate ancestor. Such an abrupt change may therefore have some consequences for nutrition.

Since the speciation from platypus, the frequency of His has apparently increased, whereas those of Thr and Gln have decreased in all other mammals (Supplemental Table S1; http://dx.doi.org/10.3168/ jds.2013-7317). Arginine is also strongly elevated in horse, showing a rapid shift from 1.6 to $4.0 \%$ since its divergence from its common ancestor with cow and dog. This shift seems unlikely to represent a simple charge constraint, because Lys, Asp, and Glu were not similarly affected. The enrichment in Ser seen in rodents was also striking ( $11.6 \%$ in rodents vs. $4.3 \%$ in primates) and seems to have occurred in the ancestor of rodents. Interestingly, we observed twice as much Met in cow as in human (2.9 vs. $1.4 \%$; Supplemental Table S1).

It has been noted that rodent taste receptors, active in the intestine and potentially involved in sensing free AA in the gut, have a much stronger response to aliphatic AA (Gly, Ala, Leu, Val, and Ile) and that human taste receptors show a greater response to Glu (Reimann et al., 2012). We noted, in $\beta$-CN (Supplemental Table $\mathrm{S} 1)$, a very slight preference for Glu in human $(6.6 \%)$ compared with rodent $(5.1 \%)$; however, the opposite trend was seen in $\alpha$-LA (Figure 1). Although it would be tempting to speculate that AA taste receptors may be optimized to sense digestion of the AA composition of the milk of their own species, the trends seen in our 
(a)

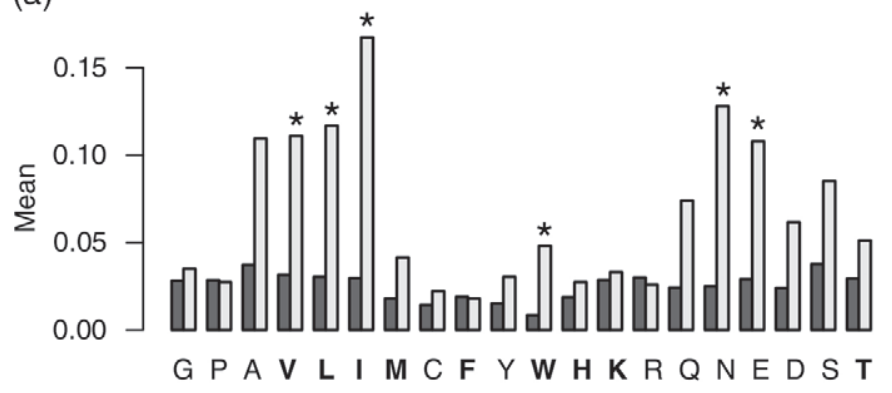

(c)

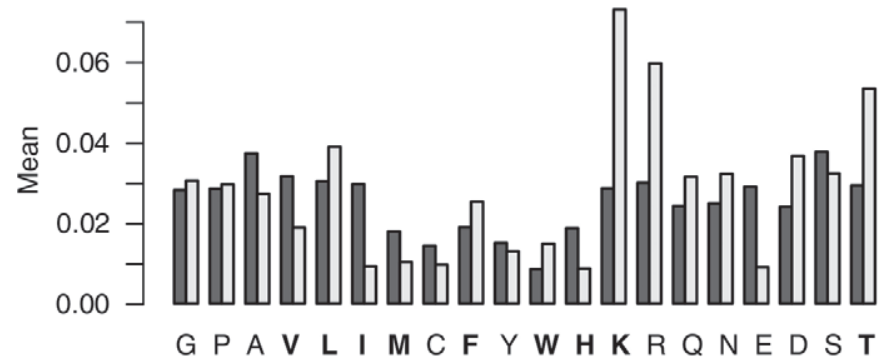

(b)

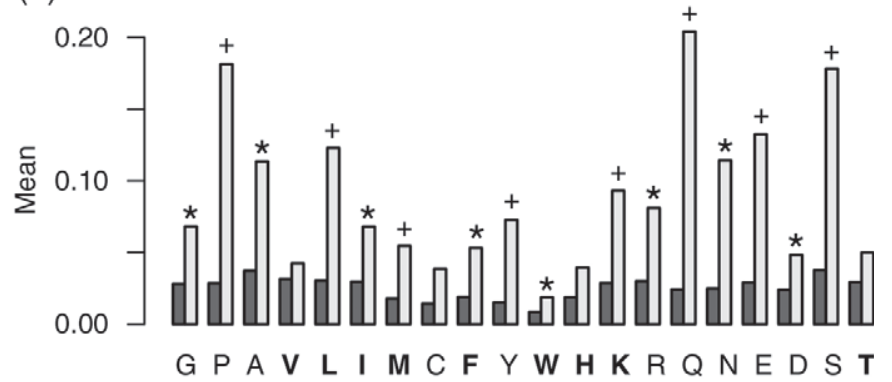

(d)

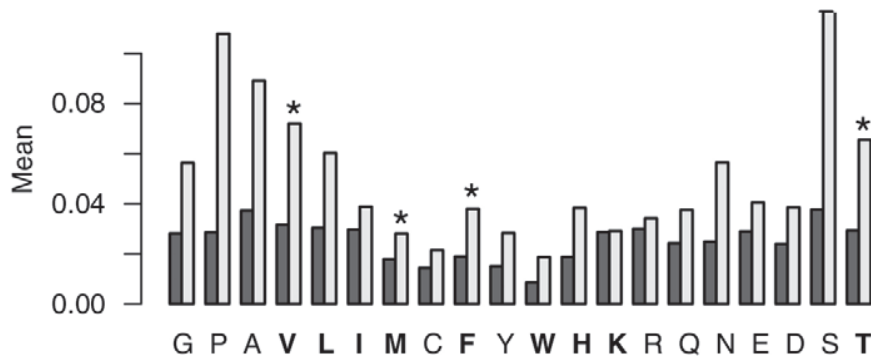

Figure 2. Comparison of the compositional changes in AA frequencies among the 4 species human, chimp, mouse, and cow between milk and nonmilk proteins: (a) $\alpha$-LA, (b) caseins $\left(\alpha_{\mathrm{S1}_{1}} \mathrm{CN}, \beta-\mathrm{CN}, \kappa-\mathrm{CN}\right)$, (c) lactoferrin, and (d) 4 non-milk-specific proteins (lactadherin, mucin, butyrophilin, and xanthine dehydrogenase). The y-axis represents the mean changes in AA frequency among the 4 species. Essential AA are presented in bold. Black bars indicate the mean change in AA frequencies for nonmilk proteins, and white bars represent that in milk proteins. The plus symbol $(+)$ above a bar indicates Bonferroni-corrected significantly over-represented AA in the milk protein(s); asterisk $(*)$ indicates a significant difference in composition before Bonferroni correction.

results are not convincing, because the aliphatic AA are generally found at equivalent or higher frequencies in human compared with rodent, contrary to this hypothesis (Figure 1, Supplemental Table S1; http://dx.doi. org/10.3168/jds.2013-7317). Nevertheless, our results clearly show several AA that show important speciesspecific or clade-specific changes in milk proteins. This led us to question whether these patterns contrasted strongly with changes in nonmilk protein.

\section{Conservation of AA Composition}

We were interested in investigating whether the differences observed for various milk proteins were significant compared with those in nonmilk proteins. Taken together, the 9 milk proteins showed much greater variation in AA content among 4 species (human, chimp, mouse and cow; see Methods) than did the nonmilk proteins (Figure 2a). The effect was most striking in $\alpha$-LA and the caseins, with less variation in the nonmilk proteins (Figure 2c and 2d). Thus, it may be that milk, although constrained for optimal AA frequencies, is under selection for very different optima in different species, resulting in evolutionary shifts in AA usage. Those AA showing an excess of change compared with nonmilk proteins are indicated by an asterisk or a plus symbol in Figure 2. Additionally, we observed equivalent change among NEAA and EAA, which suggests that questions of efficiency and optimality for particular species are not confined to EAA, but may also influence NEAA. Given the major energetic consequences of producing and digesting milk protein for both mother and child, it is not surprising that the most abundant (milk-specific) milk proteins appear to be under selective pressures. The results showed a clear distinction between non-milk-specific proteins and milk-specific proteins, with the latter showing a greater degree of compositional change for both EAA and NEAA.

A substantial cost of making a protein results from the cost of synthesizing or obtaining its individual AA. Pressure on highly expressed proteins to reduce synthesis cost is referred to as cost selection and has been demonstrated in several different organisms (Akashi and Gojobori, 2002; de Bivort et al., 2009; Vilaprinyo et al., 2010). If concentration changes are driving change in AA composition in milk proteins, then we would expect such changes to be strongest among the AA that are the most costly to synthesize (i.e., the aromatic AA Phe, Tyr, and Trp). $\alpha$-Lactalbumin is a protein that has most likely changed in concentration during the evolution of mammals, given the difference in its 
concentration between human and cow milks. Although Trp is involved in important changes in this protein (Figure 2), Phe and Tyr are not singled out compared with other AA. For this reason, we believe that concentration alone is not the sole driver of change in the AA composition between mammals and that nutritional function likely plays a major role.

We did not have detailed information on the extent of phosphorylation of caseins in different species. Phosphorylation plays a critical role in the calcium content of milk. Serine phosphate was not represented in our sequence data for any of the caseins and thus we cannot directly evaluate adaptive pressures on phosphorylated residues of caseins. However, Ser was among the 6 AA showing significant compositional changes in caseins (Figure 2). This suggests that Ser may be under adaptive selection, which could well relate to its association with phosphopeptides and consequently calcium uptake in the neonate.

Many factors might explain the significant changes in AA composition in milk proteins; for example, hydrophobicity. For many of the most hydrophobic AA (i.e., Cys, Val, Leu, Ile, Met, Trp, Phe; according to Betts and Russell, 2003), we observed a significantly higher frequency of change across mammals in both the caseins and $\alpha$-LA compared with nonmilk proteins (Figure 2). Conversely, lactoferrin did not present any significant change in these AA (Figure 2c). It could be argued that such changes in hydrophobicity could be driven by adaptive alterations that seek to change the structure and function of these proteins; for example, a change in the lactose production caused by structural changes in $\alpha$-LA. However, we have no evidence that structural changes in this protein are required to modulate its influence on lactose production. We therefore favor the argument that such compositional changes may be more likely to reflect adaptation to different nutritional requirements, but we cannot rule out the possibility of selection pressure to change the structure and function of these proteins as well.

Another factor that might drive this AA change is the degree of digestibility of the milk proteins. For example, we expected that an observed increase in Lys and Arg would correlate with greater susceptibility to pepsin digestion, a known mammalian digestive enzyme. We observed that, generally, in mammals Lys and Arg did not present any significant frequency change in $\alpha$-LA compared with nonmilk proteins (Figure 2a). Nevertheless, we observed some differences in AA frequencies that, even though they were not significant, may affect digestibility. For example, we observed a lower frequency of Lys in cat than dog (see Figure 1). The greatest change in frequency in Lys and Arg among milk proteins was observed for the caseins (significant for both residues; see Figure 2b), suggesting a greater susceptibility of these proteins to pepsin digestion in mammals. Here, it is hard to distinguish between cause and effect. Although variation in AA composition might be driving differences in potential digestibility (especially because other AA were also significantly variable), it is possible that the reverse might be true.

\section{AA in Human Milk Proteins}

To identify which AA were most enriched in human milk proteins, we compared the frequencies of each of the $20 \mathrm{AA}$ in milk proteins against the frequencies found in other human proteins (human nonmilk proteins; see Methods). Again for this analysis, we maintained the 4 groupings for the milk-specific proteins from above: $\alpha$-LA (Figure 3a), caseins (Figure 3b), lactoferrin (Figure 3c), and non-milk-specific proteins (lactoferrin, lactadherin, mucin, butyrophilin, and xanthine dehydrogenase; Figure 3d).

We found a clear distinction between the milk-specific (caseins and $\alpha$-LA) and non-milk-specific proteins. Non-milk-specific proteins did not display excess enrichment of any AA (Figure 3c), whereas milk-specific proteins did so for several AA (Figure $3 \mathrm{a}$ and $2 \mathrm{~b}$ ). We observed a considerable proportion of Ile, Cys, and Asp in $\alpha$-LA, and of Pro, Tyr, and Gln in caseins $(P=$ 0.04 for Ile, Cys, and Asp in $\alpha-\mathrm{LA} ; P=0.006, P=$ 0.02 , and $P=0.005$ for Pro, Tyr, and Gln in caseins, respectively; see Figure 3b). Our results also indicated substantial under-representation of some AA in milkspecific proteins. $\alpha$-Lactalbumin was depleted in Val and $\operatorname{Arg}(P=0.04)$ and the caseins depleted in Gly, Phe, Trp, and Asp $(P=0.001, P=0.02, P=0.02$, and $P=0.01$, respectively). Similarly, the non-milk-specific proteins displayed a reduction in Met and $\operatorname{Gln}(P=0.02$ and $P=0.01$, respectively). Additionally, our results showed a lack of distinction between the enrichment of EAA versus NEAA. Of the AA found to be enriched, 3 (Ile, Cys, and Tyr) are considered essential for infants, whereas 3 are not (Asp, Pro, and Gln). Thus, human milk proteins were not preferentially enriching for EAA compared with nonmilk proteins.

These results indicate that caseins (Figure 3b) may have primarily a nutritional role, whereas the other proteins may possess alternative primary functionalities that prevail over their nutritional role (non-milk-specific proteins and lactoferrin, Figure 3c; lactadherin, mucin, butyrophilin, and xanthine dehydrogenase, Figure 3e). Indeed, the antibacterial and antiviral activities of lactoferrin and mucin are widely known (Yolken et al., 1992; Weinberg, 2001). If the 9 major milk proteins were found in equal concentrations in milk, the nutritional quality of milk may well be quite poor. However, 
(a)

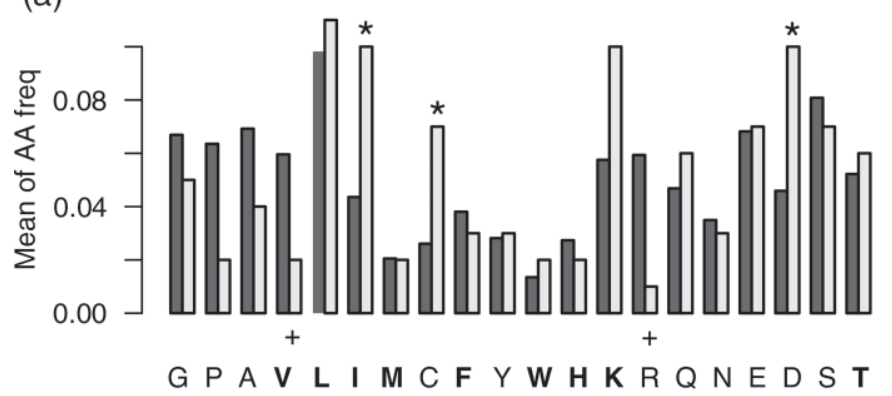

(c)

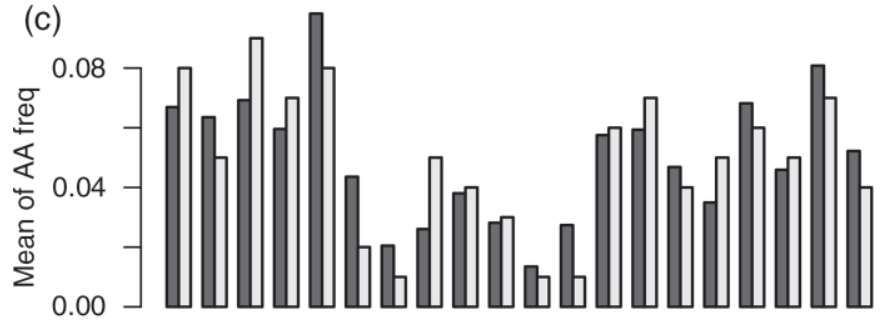

G P A V L I M C F Y W H K R Q N E D S T (b)

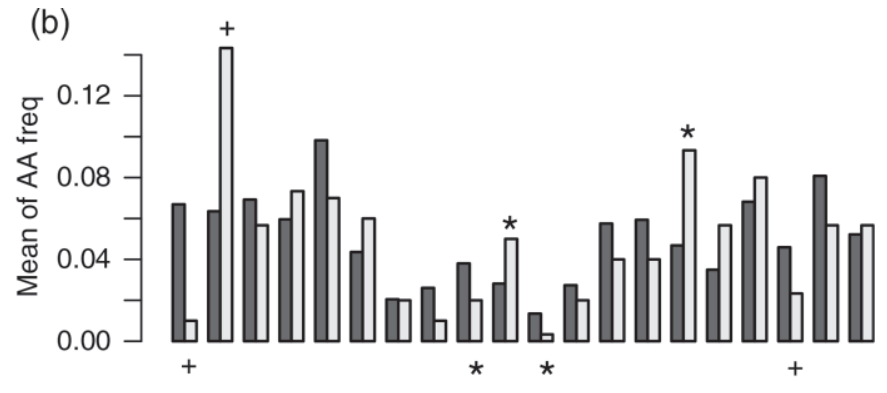

GPA V L I M C F Y W H K R Q N E D S T

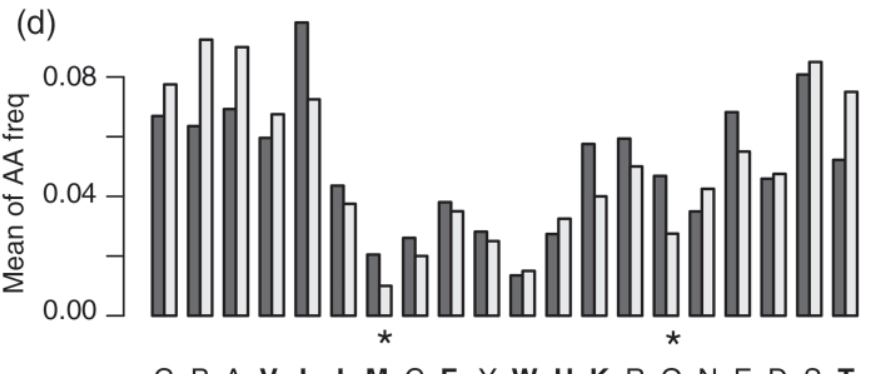

Figure 3. Comparison of the AA composition of human milk and nonmilk proteins: (a) $\alpha-\mathrm{LA}$, (b) caseins $\left(\alpha_{\mathrm{S} 1}-\mathrm{CN}, \beta-\mathrm{CN}, \kappa-\mathrm{CN}\right),(\mathrm{c})$ lactoferrin, and (d) 4 non-milk-specific proteins (lactadherin, mucin, butyrophilin, and xanthine dehydrogenase). The y-axis represents the mean changes in AA frequency (freq) among the 4 species. Essential AA are presented in bold. Black bars indicate the mean change in AA frequencies for nonmilk proteins, and white bars represent that in milk proteins. The plus symbol $(+)$ above (under) a bar indicates Bonferroni-corrected significantly over-represented (under-represented) AA in the milk protein(s); asterisk ${ }^{*}$ ) indicates a significant difference in composition before Bonferroni correction.

as seen here, milk-specific proteins are more enriched in some AA than non-milk-specific proteins (see Figure $3)$.

\section{Differences in AA Composition Between Human and Cow}

A great deal of effort is put into "humanizing" milk formulas; this is mainly achieved by adjusting the caseinto-whey ratio to mimic that of human milk. We have shown above that significant differences exist between the milks of human, chimp, mouse, and cow. Therefore, we wanted to investigate if significant differences exist in the AA composition of human milk proteins versus bovine milk proteins compared with the differences observed over the full genome of human versus cow. More importantly, we wanted to know which AA exhibited the greatest degree of change between human and cow. To address this question, we subtracted the frequency of each AA in human from its frequency in cow. We carried out this approach first for all milk proteins and second for all nonmilk proteins (see Methods).

Differences between human and bovine milk AA composition are illustrated in Figure 4 by variation in the length of the bars and their negative or positive value (positive $=$ human frequency $>$ cow frequency).
The means of the differences of the AA frequencies for nonmilk proteins (black bars) were very small and close to zero, whereas the milk proteins, represented by white bars, typically had means far from 0 (higher or lower means).

Four AA in $\alpha$-LA showed considerable variation between human and cow (Val, Ile, Trp, and Asn, all at $P$ $=0.04$; Wilcoxon test; Figure 4a). Of these AA, 3 are considered essential, with 2 of these being enriched in cow compared with human (Val and Trp). In the caseins, 7 AA displayed a substantial difference between human and cow (Phe: $P=0.002$, Lys: $P=0.01$, Tyr: $P$ $=0.004$, Glu: $P=0.002$, Ala: $P=0.01$, Asn: $P=0.04$, and Gly: $P=0.005$; Figure $4 \mathrm{~b}$ ). Both Phe and Lys, which are EAA, were present at lower levels in human compared with cow, whereas the opposite was observed for Tyr, which, interestingly, is considered an EAA for premature infants. Lactoferrin had the largest number of AA (a total of 9) exhibiting important differences between human and cow (Figure 4c), 6 of which were essential (Val, Leu, Phe, Tyr, Lys, and Thr; all $P=$ 0.04; Figure 4c). However, only 2 EAA were enriched in human compared with cow (Val and Phe; Figure 4c). Despite relevant compositional variations between human and cow milk proteins, these differences did not distinguish between EAA and NEAA. 
(a)

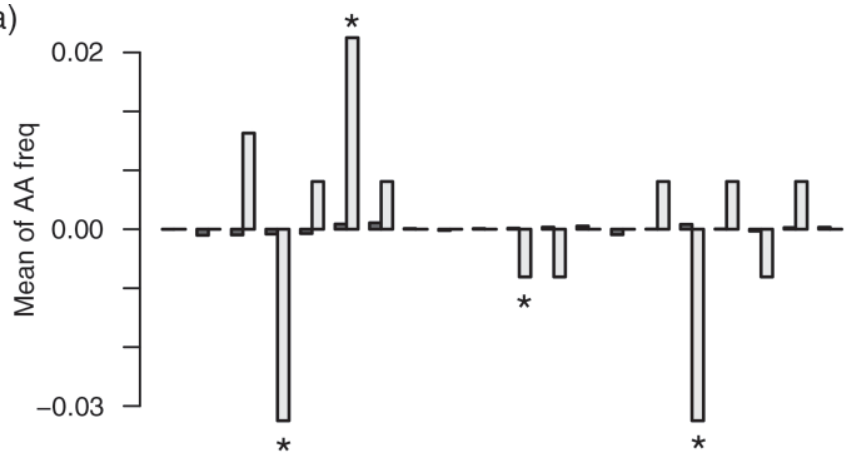

GPA V L I M C F Y W H K R Q NED S T

(c)

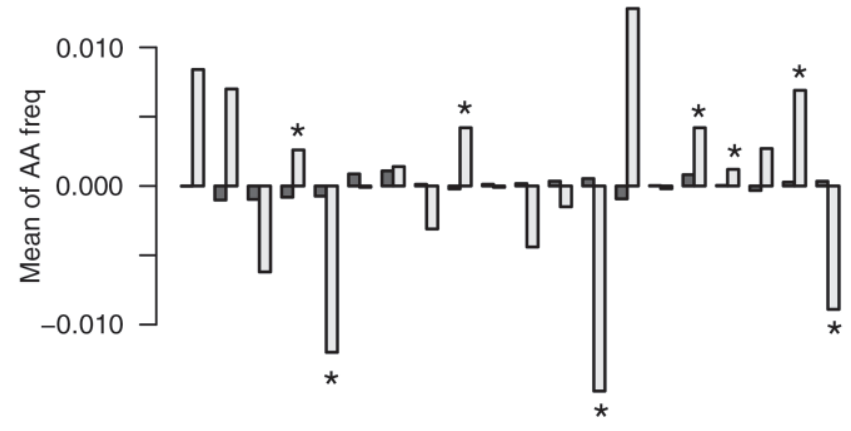

G P A V L I M C F Y W H K R Q N E D S T (b)

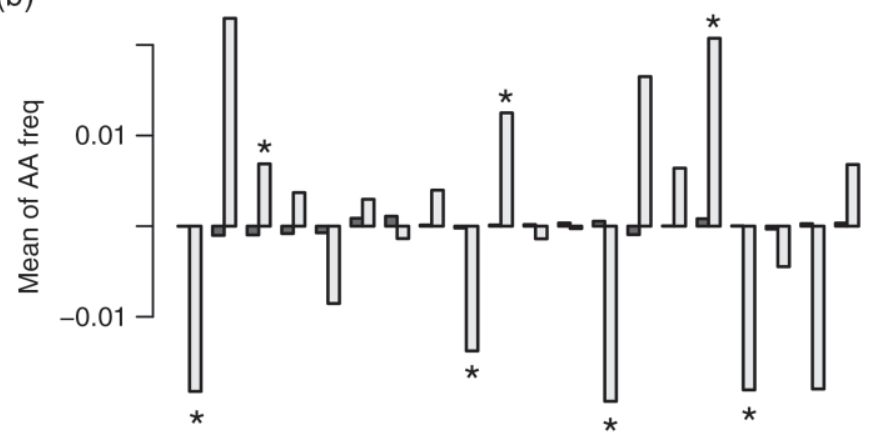

GPA V L I M C F Y W H KR Q NED S T

(d)

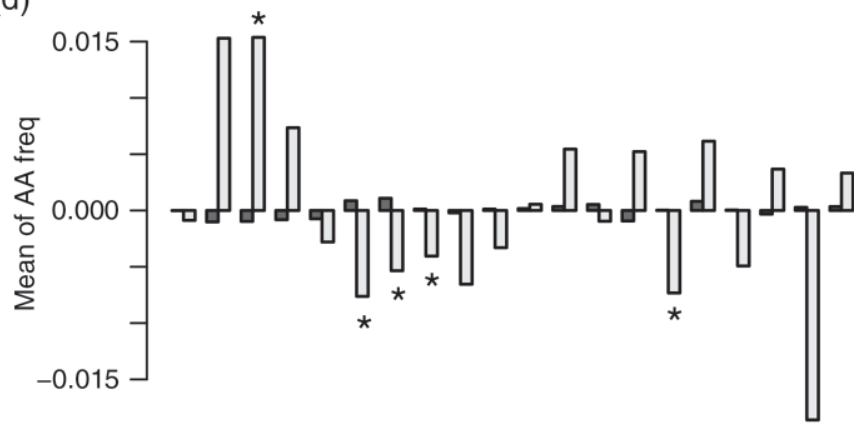

G P A V L I M C F Y W H K R Q NED S T

Figure 4. Barplot of the mean of the differences between human and cow AA composition. Positive bars indicate higher presence of an AA in human, whereas negative bars indicate higher presence in cow. The length of the bars indicates the importance of the difference between human and cow. The closer the bars are to 0, the more similar the composition of that AA is in human and cow. Essential AA are presented in bold. Black bars represent the mean AA frequencies (freq) for nonmilk protein, and white bars represent milk proteins. (a) $\alpha$-Lactalbumin; (b) caseins $\left(\alpha_{\mathrm{S} 1}-\mathrm{CN}, \beta-\mathrm{CN}, \kappa-\mathrm{CN}\right)$, (c) lactoferrin, and (d) 4 non-milk-specific proteins (lactadherin, mucin, butyrophilin, and xanthine dehydrogenase). The asterisk $(*)$ indicates a significant difference in composition before Bonferroni correction.

A noticeable amount of variation in non-milk-specific proteins was observed (see Figure 4c and 4d). However, because of the low concentrations in milk, such differences are less likely to affect nutrition than changes in the more abundant milk-specific proteins. The consequence of compositional variation in non-milk-specific proteins might be more functional in nature, promoting, for example, differences in interacting partners or bioactivity (Khaldi and Shields, 2011). As such, the changes observed for lactoferrin may be associated with its antimicrobial activity, influencing adaptations in specificity for certain microbes that are unique to human or cow.

Of the total 18 AA presenting differences in the caseins and $\alpha$-LA, 12 displayed changes in opposing directions, such that in human, caseins were rich in a particular AA that was concurrently depleted in human $\alpha$-LA (and vice versa). This was seen most strongly in the following AA: Val, Ile, Trp, Asn, Gly, Phe, Tyr, Lys, and Glu. Because caseins and $\alpha$-LA constitute most of the proteins in the milk, compositional differences in these proteins are relevant to nutrition. It is possible that when the caseins are depleted in a certain AA, $\alpha$-LA will compensate for that depletion. This may be due to the different natures of these proteins; caseins, despite containing significant secondary structures that may help mediate casein-casein interactions (Farrell et al., 2013), are considered disordered proteins, whereas $\alpha$-LA is more structured, with the AA composition of disordered proteins being distinct from that of structured proteins (Dunker et al., 2001).

The differences in the AA composition of human and bovine milk demonstrate that efforts aimed at adjusting the protein concentration of milk formulas do not alleviate all variation between milks of the species. Single AA may be added to compensate for such AA differences, but will not compensate for the missing diand tripeptides that are composed of these AA. Such 
di- and tripeptides might be important for signaling or several alternative bioactivities, which may be lost in bovine milk.

\section{Evolutionary Constraints}

Milk plays an irreplaceable role in the development of young mammals. It carries the fats, sugars, and AA required for normal and healthy growth. We showed that the pressure exerted by millions of years of co-evolution between milk and the neonate's nutritional needs has enriched and depleted certain AA in different lineages (Figures 1-4). Compared with the 4 major milk-specific proteins, the 5 non-milk-specific proteins did not display an important enrichment in EAA (Figure 3-4). Because these non-milk-specific proteins also occur in milk, but at much lower abundances, their primary role may not be nutritional, limiting the advantage of modulating their AA content during evolution. Aside from their nutritional role, numerous functional roles are additionally ascribed to milk-specific proteins. This balance between functional and nutritional duties might be a major contributor in the decision to enrich or deplete particular AA in certain milk proteins.

The balance of energy contributed by the mother to her child, in terms of the quality of milk she provides, represents an important area for evolutionary optimization. Over-investment of the mother's EAA in her milk (or those AA that require more energy to synthesize) will reduce her own future evolutionary fitness, thus raising the question of whether milk is truly optimal for infant nutrition in certain species. This might explain, in part, why marked changes in composition occur during evolution, as the evolutionary niche occupied by a species alters the optimal balance of resources committed to the offspring. This has consequences for the optimal design of milk formulas for infants at different stages of development. Indeed, some of the enriched AA in human may be cleaved to produce di- and tripeptides with fundamental bioactivities, which cannot be replaced by enriching milk formulas with single AA.

\section{CONCLUSIONS}

The species-specific adaptation of milk to the nutritional requirements of the neonate may involve multiple processes, including the gain and loss of genes encoding the proteins, changes in expression levels of the proteins, and changes in composition of the proteins themselves. We chose to focus on the direct evolutionary comparison of protein composition as it can be readily compared between diverse species because of the data currently available. Variation in the total protein concentration and the distinct whey-to-casein ratio of the milk of a species are central to its tailored nutritional value. Here, our systematic study of milk proteins demonstrates for the first time that variation in AA composition may also play a contributory role in nutritional adaptation. This adaptation may encompass a whole series of factors relevant to the nutritional benefit of the milk proteins. These may include digestibility, hydrophobicity, and charge factors influencing transit through the gut, and binding of calcium and other nutrients, as well as the spectrum of AA made available for protein synthesis in the neonate.

\section{ACKNOWLEDGMENTS}

This work was supported by the Irish Research Council for Science, Engineering and Technology, co-funded by Marie Curie Actions under FP7; and by Enterprise Ireland Grant Number CC20080001 (Food for Health Ireland). We thank Gavin Conant (University of Missouri, Columbia) for his helpful and valuable comments. We also thank Dick Fitzgerald (University of Limerick, Castletroy, Co. Limerick, Ireland) and Fergal Casey (Novartis Institute of Biomedical Research, Emeryville, CA) for useful discussion.

\section{REFERENCES}

Akashi, H., and T. Gojobori. 2002. Metabolic efficiency and amino acid composition in the proteomes of Escherichia coli and Bacillus subtilis. Proc. Natl. Acad. Sci. USA 99:3695-3700.

Altschul, S. F., T. L. Madden, A. A. Schaffer, J. Zhang, Z. Zhang, W. Miller, and D. J. Lipman. 1997. Gapped BLAST and PSI-BLAST: A new generation of protein database search programs. Nucleic Acids Res. 25:3389-3402.

Benton, M. J., and P. C. Donoghue. 2007. Paleontological evidence to date the tree of life. Mol. Biol. Evol. 24:26-53.

Betts, M. J., and R. B. Russell. 2003. Amino acid properties and consequences of substitutions. Pages 289-316 in Bioinformatics for Geneticists. M. R. Barnes and I. C. Gray, ed. John Wiley \& Sons Ltd., Chichester, UK.

Bevilacqua, C., J. C. Helbling, G. Miranda, and P. Martin. 2006. Translational efficiency of casein transcripts in the mammary tissue of lactating ruminants. Reprod. Nutr. Dev. 46:567-578.

Bordin, G., F. Cordeiro Raposo, B. de la Calle, and A. R. Rodriguez. 2001. Identification and quantification of major bovine milk proteins by liquid chromatography. J. Chromatogr. A 928:63-76.

Burditt, L. J., D. Parker, R. K. Craig, T. Getova, and P. N. Campbell. 1981. Differential expression of alpha-lactalbumin and casein genes during the onset of lactation in the guinea-pig mammary gland. Biochem. J. 194:999-1006.

Davis, T. A., H. V. Nguyen, R. Garcia-Bravo, M. L. Fiorotto, E. M. Jackson, D. S. Lewis, D. R. Lee, and P. J. Reeds. 1994. Amino acid composition of human milk is not unique. J. Nutr. 124:1126-1132.

de Bivort, B. L., E. Perlstein, S. Kunes, and S. Schreiber. 2009. Amino acid metabolic origin as an evolutionary influence on protein sequence in yeast. J. Mol. Evol. 68:490-497.

Dunker, A. K., J. D. Lawson, C. J. Brown, R. M. Williams, P. Romero, J. S. Oh, C. J. Oldfield, A. M. Campen, C. M. Ratliff, K. W. Hipps, J. Ausio, M. S. Nissen, R. Reeves, C. Kang, C. R. Kissinger, R. W. Bailey, M. D. Griswold, W. Chiu, E. C. Garner, and Z. Obradovic. 2001. Intrinsically disordered protein. J. Mol. Graph. Model. 19:26-59. 
Farrell, H. M., Jr., E. M. Brown, and E. L. Malin. 2013. Higher order structures of the caseins: A paradox? Pages 161-184 in Advanced Dairy Chemistry. P. L. H. McSweeney and P. F. Fox, ed. Springer, New York, NY.

Farrell, H. M., R. Jimenez-Flores, G. T. Bleck, E. M. Brown, J. E. Butler, L. K. Creamer, C. L. Hicks, C. M. Hollar, K. F. Ng-KwaiHang, and H. E. Swaisgood. 2004. Nomenclature of the proteins of cows' milk-Sixth revision. J. Dairy Sci. 87:1641-1674.

Fernstrom, J. D. 1983. Role of precursor availability in control of monoamine biosynthesis in brain. Physiol. Rev. 63:484-546.

Fürst, P., and P. Stehle. 2004. What are the essential elements needed for the determination of amino acid requirements in humans? J. Nutr. 134:1558S-1565S

Ha, E., and M. B. Zemel. 2003. Functional properties of whey, whey components, and essential amino acids: Mechanisms underlying health benefits for active people. J. Nutr. Biochem. 14:251-258.

Hambræus, L., and B. Lönnerdal. 2003. Nutritional Aspects of Milk Proteins. Pages 605-645 in Advanced Dairy Chemistry-1: Proteins. P. F. Fox and P. L. H. McSweeney, ed. Springer, New York, NY.

Hui, Y. H., R. C. Chandan, S. Clark, N. Cross, J. C. Dobbs, W. J. Hurst, E. B. Smith, L. M. Nollet, E. Shimoni, and N. Sinha. 2007. Handbook of Food Products Manufacturing. John Wiley \& Sons Inc., New York, NY.

Jenness, R. 1974a. Biosynthesis and composition of milk. J. Invest. Dermatol. 63:109-118.

Jenness, R. 1974b. Composition of milk. Pages 3-107 in Lactation: A Comprehensive Treatise. Vol. III. Nutrition and Biochemistry of Milk Maintenance. B. L. Larson and V. R. Smith, ed. Academic Press, New York, NY.

Jenness, R. 1986. Lactational performance of various mammalian species. J. Dairy Sci. 69:869-885.

Jensen, R. G. 1995. Handbook of Milk Composition. Academic Press, San Diego, CA.

Khaldi, N., and D. C. Shields. 2011. Shift in the isoelectric-point of milk proteins as a consequence of adaptive divergence between the milks of mammalian species. Biol. Direct 6:40.

Lemay, D. G., D. J. Lynn, W. F. Martin, M. C. Neville, T. M. Casey, G. Rincon, E. V. Kriventseva, W. C. Barris, A. S. Hinrichs, A. J. Molenaar, K. S. Pollard, N. J. Maqbool, K. Singh, R. Murney, E. M. Zdobnov, R. L. Tellam, J. F. Medrano, J. B. German, and M. Rijnkels. 2009. The bovine lactation genome: Insights into the evolution of mammalian milk. Genome Biol. 10:R43.

Lönnerdal, B. 2003. Nutritional and physiologic significance of human milk proteins. Am. J. Clin. Nutr. 77:1537S-1543S.

Martin, P., C. Cebo, and G. Miranda. 2013. Interspecies comparison of milk proteins: Quantitative variability and molecular diversity. Pages 387-429 in Advanced Dairy Chemistry. P. L. H. McSweeney and P. F. Fox, ed. Springer, New York, NY.

Martin, P., P. Ferranti, C. Leroux, and F. Addeo. 2003. Non-bovine caseins: Quantitative variability and molecular diversity. Pages 277-317 in Advanced Dairy Chemistry - 1 Proteins. P. F. Fox and P. L. H. McSweeney, ed. Springer, New York, NY.

Meredith, R. W., J. E. Janecka, J. Gatesy, O. A. Ryder, C. A. Fisher, E. C. Teeling, A. Goodbla, E. Eizirik, T. L. L. Simao, T. Stadler, D. L. Rabosky, R. L. Honeycutt, J. J. Flynn, C. M. Ingram, C. Steiner, T. L. Williams, T. J. Robinson, A. Burk-Herrick, M. Wes- terman, N. A. Ayoub, M. S. Springer, and W. J. Murphy. 2011. Impacts of the cretaceous terrestrial revolution and $\mathrm{KPg}$ extinction on mammal diversification. Science 334:521-524.

Nelson, M. M., and H. M. Evans. 1958. Sulfur amino acid requirement for lactation in the rat. Proc. Soc. Exp. Biol. Med. 99:723-725.

Parry, G., J. Li, J. Stubbs, M. J. Bissell, C. Schmidhauser, A. P. Spicer, and S. J. Gendler. 1992. Studies of Muc-1 mucin expression and polarity in the mouse mammary gland demonstrate developmental regulation of Muc-1 glycosylation and establish the hormonal basis for mRNA expression. J. Cell Sci. 101:191-199.

Permyakov, E. A., and L. J. Berliner. 2000. $\alpha$-Lactalbumin: Structure and function. FEBS Lett. 473:269-274.

Petersen, T. N., S. Brunak, G. von Heijne, and H. Nielsen. 2011. SignalP 4.0: Discriminating signal peptides from transmembrane regions. Nat. Methods 8:785-786.

Reeds, P. J. 2000. Dispensable and indispensable amino acids for humans. J. Nutr. 130:1835S-1840S.

Reimann, F., G. Tolhurst, and F. M. Gribble. 2012. G-protein-coupled receptors in intestinal chemosensation. Cell Metab. 15:421-431.

Schaechter, J. D., and R. J. Wurtman. 1990. Serotonin release varies with brain tryptophan levels. Brain Res. 532:203-210.

Shewale, J. G., S. K. Sinha, and K. Brew. 1984. Evolution of alphalactalbumins. The complete amino acid sequence of the alpha-lactalbumin from a marsupial (Macropus rufogriseus) and corrections to regions of sequence in bovine and goat alpha-lactalbumins. J. Biol. Chem. 259:4947-4956.

Snyderman, S. E., A. Boyer, and L. E. Holt Jr. 1961. Evaluation of protein foods in premature infants. Page 331 in Meeting Protein Needs of Infants and Children. National Academy Press, Washington, DC.

Thompson, J. D., D. G. Higgins, and T. J. Gibson. 1994. CLUSTAL $\mathrm{W}$ : Improving the sensitivity of progressive multiple sequence alignment through sequence weighting, position-specific gap penalties and weight matrix choice. Nucleic Acids Res. 22:4673-4680.

Valenti, P., and G. Antonini. 2005. Lactoferrin: An important host defence against microbial and viral attack. Cell. Mol. Life Sci. 62:2576-2587.

Véron, P., E. Segura, G. Sugano, S. Amigorena, and C. Thery. 2005. Accumulation of MFG-E8/lactadherin on exosomes from immature dendritic cells. Blood Cells Mol. Dis. 35:81-88.

Vilaprinyo, E., R. Alves, and A. Sorribas. 2010. Minimization of biosynthetic costs in adaptive gene expression responses of yeast to environmental changes. PLOS Comput. Biol. 6:e1000674.

Weinberg, E. D. 2001. Human lactoferrin: A novel therapeutic with broad spectrum potential. J. Pharm. Pharmacol. 53:1303-1310.

Yang, Z. 2007. PAML 4: Phylogenetic analysis by maximum likelihood. Mol. Biol. Evol. 24:1586-1591.

Yang, Z., S. Kumar, and M. Nei. 1995. A new method of inference of ancestral nucleotide and amino acid sequences. Genetics 141:1641-1650.

Yolken, R. H., J. A. Peterson, S. L. Vonderfecht, E. T. Fouts, K. Midthun, and D. S. Newburg. 1992. Human milk mucin inhibits rotavirus replication and prevents experimental gastroenteritis. J. Clin. Invest. 90:1984-1991.

Zotter, S., P. C. Hageman, A. Lossnitzer, W. J. Mooi, and J. Hilgers. 1988. Tissue and tumor distribution of human polymorphic epithelial mucin. Cancer Rev. 11-12:55-101. 